\title{
Neoadjuvant Imatinib in Locally Advanced Gastrointestinal Stromal Tumors of the Stomach: Report of Three Cases
}

\author{
Ji Seon Oh, M.D. ${ }^{1}$, Jae-Lyun Lee, M.D. ${ }^{1}$, Mi-Jung Kim, M.D. ${ }^{2}$, Min-Hee Ryu, M.D. ${ }^{1}$, Heung Moon Chang, \\ M.D. ${ }^{1}$, Tae Won Kim, M.D. ${ }^{1}$, Se Jin Jang, M.D. ${ }^{2}$, Jeong Hwan Yook, M.D. ${ }^{3}$, Sung Tae Oh, M.D. ${ }^{3}$, Byung \\ Sik Kim, M.D. ${ }^{3}$ and Yoon-Koo Kang, M.D. ${ }^{1}$ \\ Departments of ${ }^{1}$ Internal Medicine, ${ }^{2}$ Pathology, ${ }^{3}$ Surgery, Asan Medical Center, University of Ulsan College of \\ Medicine, Seoul, Korea
}

Neoadjuvant imatinib therapy used to treat locally advanced or metastatic gastrointestinal stromal tumors (GI ST) remains under active investigation. We studied three cases of locally advanced gastric GISTs treated with imatinib on a neoadjuvant basis, followed by a complete surgical resection. Three patients were diagnosed with locally advanced unresectable GIST of the stomach and were started on imatinib $400 \mathrm{mg} /$ day. After the imatinib treatment, partial responses were achieved in all patients and the tumors were considered resectable. Surgical resection was done after 7,11 , and 8 months of imatinib therapy, respectively. In one case, a metastatic liver lesion was detected during the imatinib treatment using computed tomography scans, so the imatinib therapy was maintained for 11 months postoperatively. In the other

\section{INTRODUCTION}

Gastrointestinal stromal tumors (GISTs), although rare overall, are the most common mesenchymal tumors of the gastrointestinal tract. The stomach is the most common site for GISTs (1). Morphologically, GISTs are characterized as spindle cell or mixed epithelioid cell mesenchymal tumors located within the gastrointestinal tract, and they are characterized by expression of the c-KIT protein (CD117) (1). The current definitive treatment for localized GISTs is surgical resection (2). Unresectable or metastatic GISTs have been associated with a poor prognosis, because chemotherapy and radiation therapy are ineffective in the treatment of GISTs (3). Activating mutations of $c$-kit are present in up to $88.2 \%$ of GISTs. Of the GISTs lacking c-kit mutations $35 \sim 40 \%$ have activating mutations in the related receptor tyrosine kinase gene, platelet-derived growth factor receptor a (PDGFRA). In addition, 7\% of GISTs have no detectable mutation $(4,5)$. The $c$-kit mutation has been re-

Correspondence: Yoon-Koo Kang, Division of Oncology, Department of Internal Medicine, Asan Medical Center, 388-1, Poongnap-dong, Songpa-gu, Seoul 138-736, Korea. (Tel) 82-2-3010-3210, (Fax) 822-3010-6961, (E-mail) ykkang@amc.seoul.kr

Received August 10, 2006, Accepted August 30, 2006 two patients without distant metastasis, imatinib treatment was not restarted after surgery. Mutational analysis revealed a mutation in exon 11 of the c-kit gene in two patients, and wild-type c-kit and PDGFRA in one patient. During pathology review of all three cases, we noted several features common to imatinib treatment. There was no evidence of tumor recurrence in all three patients at respective follow-up visits of 22,15 , and 7 months. These results suggest that the neoadjuvant imatinib therapy is a potentially curative approach for selected patients with locally advanced GIST. (Cancer Res Treat. 2006;38:178-183)

Key Words: Gastrointestinal stromal tumors, Imatinib, Neoadjuvant therapy, Surgery

ported to be present in $74 \%$ of Korean GIST cases (6). These gain-of-function mutations result in constitutive activation of KIT or PDGFRA without ligand stimulation and are considered to be central in the pathogenesis of GIST (1).

Imatinib mesylate (formerly STI-571; Gleevec ${ }^{\mathrm{TM}}$ in the United States, Glivec ${ }^{\mathrm{TM}}$ in other countries), a tyrosine kinase inhibitor, was shown to inhibit the kinase activities of KIT and PDGFR, as well as being effective against metastatic or unresectable GISTs (5). During the treatment of advanced GIST in phase II and phase III clinical trials of imatinib mesylate , 47 $67 \%$ of patients achieved a partial response (PR), $0 \sim 5 \%$ of patients showed a complete response (CR), and $18 \sim 32 \%$ of patients displayed stable disease (SD), based on response evaluation criteria in solid tumor (RECIST) criteria, over 6 24 months of follow-up visits $(7 \sim 10)$. Thus, up to $89 \%$ of GIST patients benefited from imatinib mesylate, which also increased overall survival $(7 \sim 10)$. In data collected from five institutions in Korea between 2001 and 2003, 61\% of patients with metastatic or unresectable GISTs achieved PR and $31 \%$ had SD, while only $8 \%$ of patients showed disease progression (11). The effectiveness of imatinib in treating metastatic GISTs led to clinical trials of this agent in patients with locally advanced GISTs, to improve response and to reduce the extent of resection, thus reducing surgery-related morbidity and functional loss. The ability of neoadjuvant imatinib to treat locally advanced or metastatic GISTs remains under investigation. Here we describe 
three patients with initially unresectable GIST of the stomach: all three tumors could be completely resected after neoadjuvant imatinib treatment.

\section{CASE REPORT}

\section{1) Case 1}

A 45-year-old female was referred from a primary clinic in August 2003 for an intraabdominal mass detected by an ultrasound. She had complained of abdominal discomfort and diarrhea during the preceding 2 weeks. There were no other gastrointestinal complaints. On physical examination, a large non-tender mass was palpable on the left upper quadrant. Initial laboratory results were unremarkable. A computed tomography (CT) scan of the abdomen with contrast enhancement revealed a 13$\mathrm{cm}$ sized, large heterogeneous enhancing, exophytic mass involving the cardia, fundus, and body of the stomach, with direct invasion into the spleen, left adrenal gland, pancreas tail, adjacent abdominal wall, and diaphragm. An ultrasound-guided core biopsy showed proliferation of spindle cells with high cellularity and frequent mitoses $(50 \sim 60 / 50 \mathrm{HPFs})$. These cells were diffusely immunoreactive for KIT and CD34, but negative for the S-100 protein and smooth muscle actin, confirming a diagnosis of GIST with a high risk of malignancy. Genotyping of exons 9, 11, 13, and 17 of $c$-kit and exons 12 and 18 of PDGFRA revealed a deletion mutation in exon 11 of the $c$-kit gene. The patient was diagnosed with unresectable GIST and started on an imatinib $400 \mathrm{mg} /$ day treatment in September 2003. Sequential CT scans were performed at $1,3,5$, and 6 months. A CT scan performed 1 month after starting imatinib showed a significant reduction in tumor size, to $7 \mathrm{~cm}$ in the long axis. At 3 months, the tumor showed no significant changes in size and extent. At 5 months, a new, tiny, and low density lesion appeared in the liver (segment IV). No further changes were detected at 6 months (Fig. 1). After about 7 months of imatinib therapy, the tumor was considered resectable and the patient underwent total gastrecto- my, splenectomy and partial hepatectomy. The resected stomach revealed an $8 \mathrm{~cm}$-submucosal mass, consisting of a few spindle cells and abundant hyalinized stroma. The immunoprofile of the spindle cells was identical to that of the previous biopsy (KIT and CD34 positive), suggesting that these cells were residual tumor cells. In contrast to the previous biopsy, the tumor was paucicellular and mitoses were rare (Fig. 4). The resected hepatic mass was entirely necrotic with no viable cells. Spleen and regional lymph nodes were free from tumor cells. One month after the surgery, post-operative imatinib therapy was restarted. Follow up CT scans at 5, 8, and 12 months showed that no residual disease was present, and imatinib therapy was then stopped. Currently, 10 months after cessation of imatinib, the patient remains in remission.

\section{2) Case 2}

In December 2003, a 49-year-old male with a 1-month history of dizziness and melena was referred from a primary clinic for anemia and a gastric mass, as shown by routine laboratory tests and esophagogastroduodenoscopy (EGD). Twenty years earlier, this patient had undergone abdominal surgery, consisting of distal gastrectomy with gastrojejunostomy, due to a gastric ulcer perforation. In addition, he was receiving thyroid hormone replacement therapy for hypothyroidism diagnosed 10 years previously. He complained of no other gastrointestinal problems, except the abdominal discomfort. Physical examination of the abdomen revealed a large, non-tender, and hard mass palpated on the left upper quadrant. Laboratory results were unremarkable, except for a hemoglobin level of $9.2 \mathrm{mg} / \mathrm{dl}$ and an albumin level of $2.9 \mathrm{~g} / \mathrm{dl}$. A CT scan performed at the local clinic in September 2003 revealed a large $(10 \mathrm{~cm}$ in long axis) irregular lobulated solid mass with necrosis in the left upper quadrant, abutting the stomach. The mass extended to the pancreas tail portion, but did not show any lymphadenopathy. Endoscopic biopsy performed at an outside hospital revealed a GIST, with cells immunopositive for KIT and CD34. Mitoses were frequent (about 5/20 HPFs) and cellularity was high. In December 2003, the patient was started
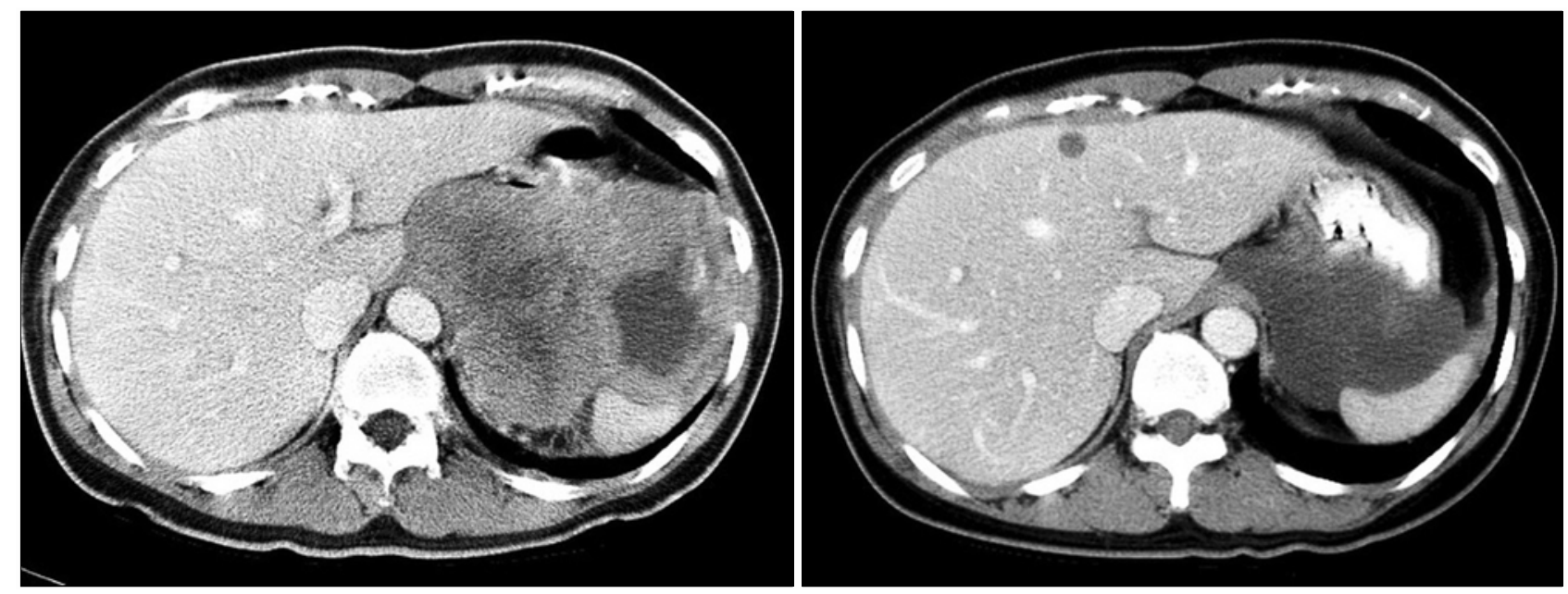

Fig. 1. Pre- and post-treatment computed tomography (CT) scans in case 1. CT scans at diagnosis (left) and after 6 months of imatinib treatment (right) are shown. A liver metastasis was detected after 5 months of treatment. 
on an imatinib mesylate treatment of $400 \mathrm{mg} /$ day. After 2 months, a CT scan showed that the mass size had been reduced to $8 \mathrm{~cm}$ in the long axis. Sequential CT scans performed 4 and 7 months after the treatment revealed further reduction in mass size, to a length of $5 \mathrm{~cm}$ in the long axis. A CT scan performed after 10 months of treatment showed no further reduction in the size of the mass and no new lesions (Fig. 2).

In November 2004, after 11 months of imatinib treatment, the patient underwent a total gastrectomy with distal pancreatectomy and splenectomy. The resected stomach revealed a 7.5 cm-submucosal mass, which was composed of a few KIT-positive tumor cells and abundant hyalinized stroma. The immunoprofile was the same as that of the previous biopsy. The residual tumor cells were pyknotic, with no identifiable mitoses (Fig. 4). The resected pancreas, spleen, and regional lymph nodes were free of tumor. Genotyping of exons $8,9,11,13,14,15$, and 17 of $c$-kit and exons 12 and 18 of PDGFRA showed no mutations in either gene. Imatinib therapy was not restarted af- ter surgery, and a follow up CT scan performed 12 months after surgery revealed no evidence of disease recurrence. Currently, 15 months after surgery, the patient shows no evidence of recurrence.

\section{3) Case 3}

A 63-year-old male with a 6-month history of dizziness and melena was referred from a local clinic in October 2004. Six months earlier, a laboratory test revealed anemia, and the patient was transfused with 2 pints of packed RBC. EGD showed no specific findings other than gastritis. One month prior to referral, his dizziness recurred. An EGD performed at the local clinic showed a gastric malignancy and an endoscopic biopsy revealed GIST. He was transfused with packed RBC at the local clinic before admission to the medical department of our hospital. At that time, he was a 40 pack-year smoker. His past medical history and physical examination were unremarkable. Laboratory results were within normal limits except for a hemo-
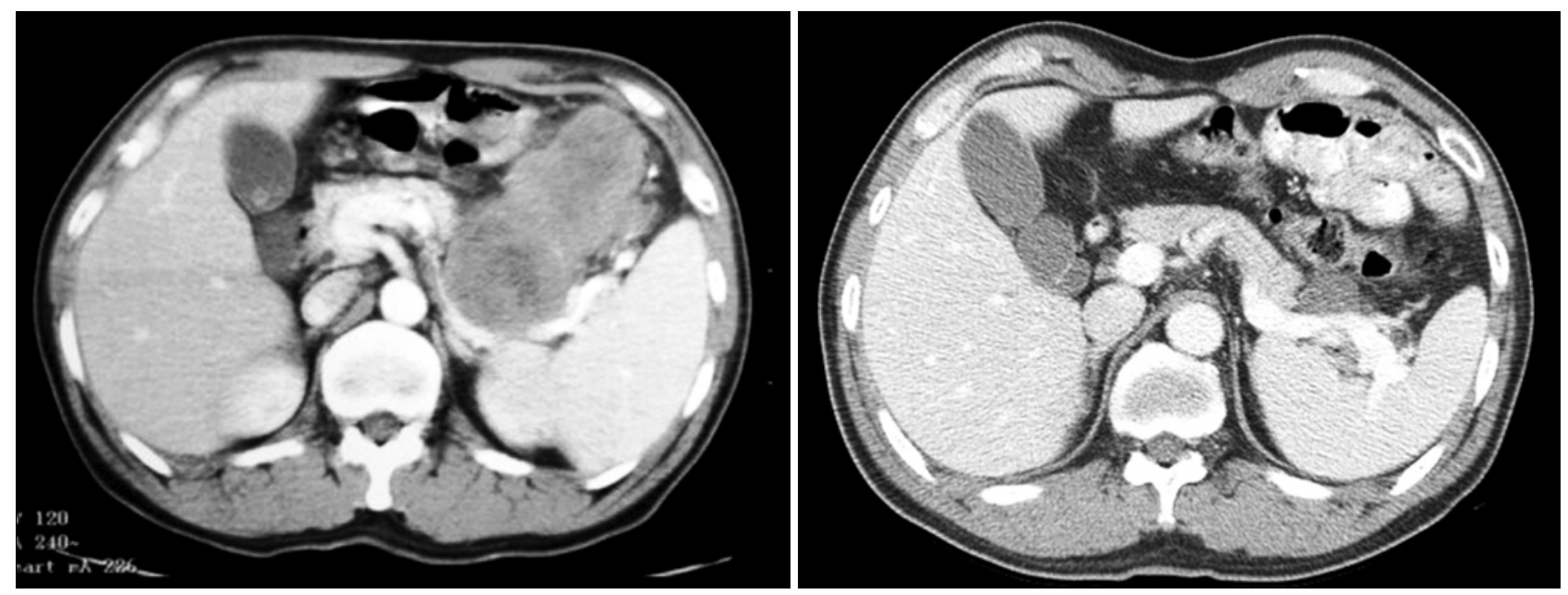

Fig. 2. Pre- and post-treatment CT scans in case 2. CT scans at diagnosis (left) and after 10 months of imatinib treatment (right) are shown.
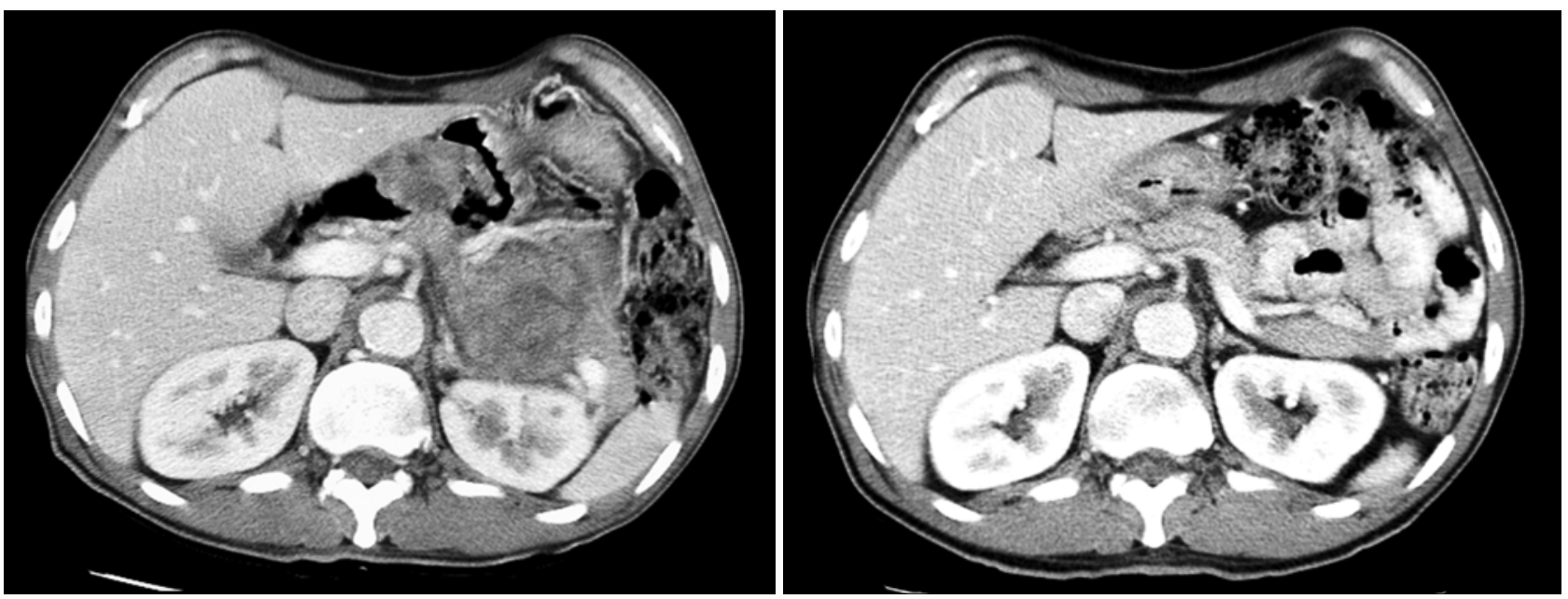

Fig. 3. Pre- and post-treatment CT scans in case 3. CT scans at diagnosis (left) and after 7 months of imatinib treatment (right) are shown. 

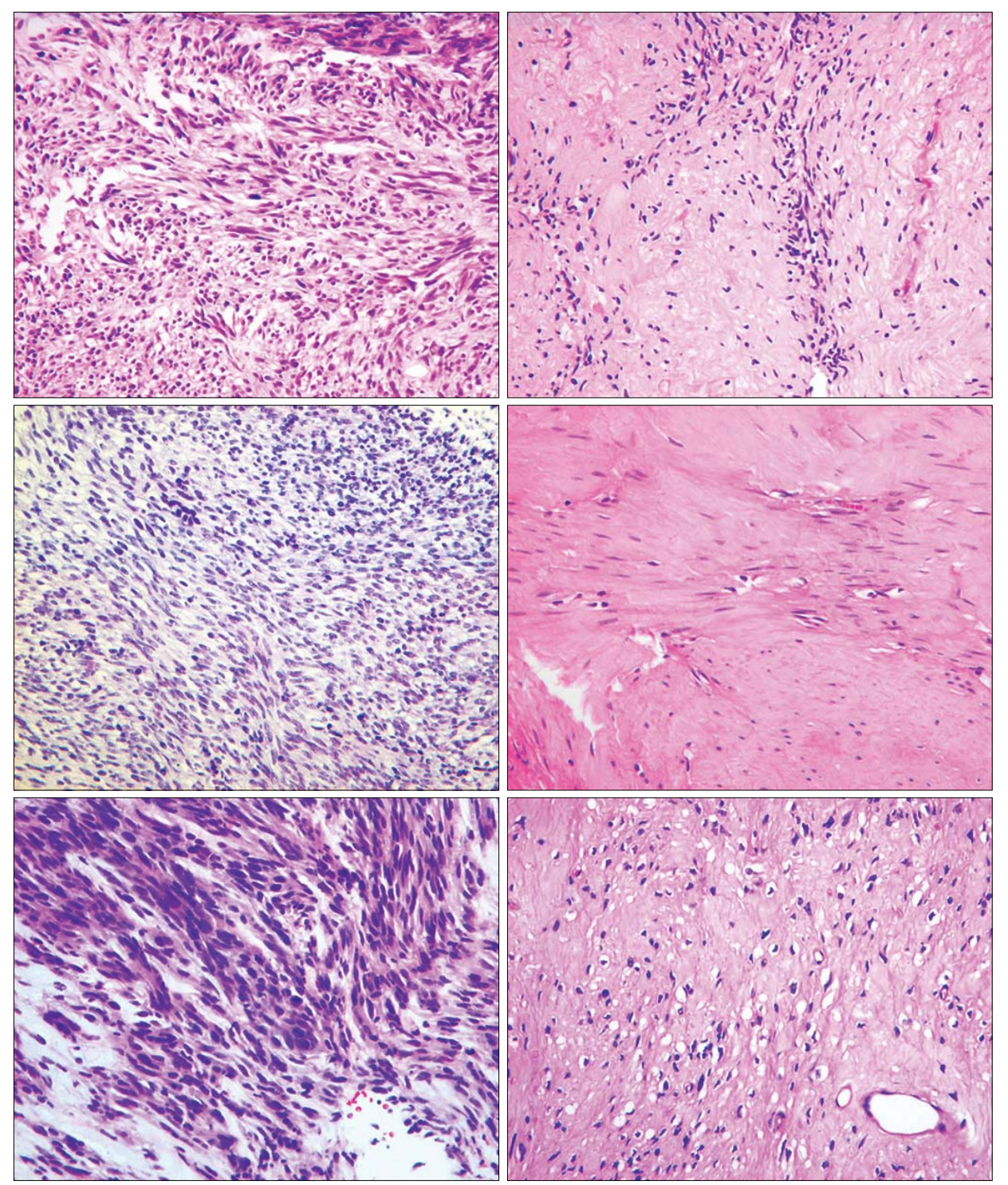

Fig. 4. Pathologic findings and changes after treatment of each patient (hematoxylin and eosin, magnification $\times 400)$. Pathologic findings before (left side) and after (right side) treatment in case 1 (upper), case 2 (middle) and case 3 (lower) are shown.

globin level of $9.8 \mathrm{mg} / \mathrm{dl}$. EGD revealed a huge ulceroinfiltrative mass over the cardia and fundus of the stomach. Histological examination of an endoscopic biopsy showed that the mass was a GIST and was positive for KIT and CD34. Mutation analyses showed a deletion mutation in exon 11 of $c$-kit, but no mutations in exons 9, 13, and 17 of c-kit and exons 12 and 18 of PDGFRA. CT scans revealed a large $\left(7.5 \times 6.9 \times 7.5 \mathrm{~cm}^{3}\right)$ low attenuated mass involving the gastroesophageal junction, cardia, and body of the stomach. There was no evidence of distant metastases, but a pancreatic tail invasion was suspected, excluding curative resection. Beginning in October 2004, the patient was started on an imatinib therapy of $400 \mathrm{mg} /$ day. After 1.5 months, the size of the mass was reduced to $5.5 \mathrm{~cm}$ in the long axis, which was again reduced at 4.5 months to $4.5 \mathrm{~cm}$. At 7 months, there were no further reductions in the mass size and no changes in extent of the disease (Fig. 3). In June 2005, after 8 months of imatinib use, the patient underwent a total gastrectomy. The resected specimen showed an ill-defined, 6$\mathrm{cm}$ mass involving the cardia. The post-treatment histologic find- ings were similar to those described for cases 1 and 2 (Fig. 4, Table 1). Imatinib therapy was not restarted after surgery, and a follow up CT scan 3.5 months after surgery showed no evidence of local recurrence or distant metastases. Currently, 7 months after surgery, the patient shows no evidence of disease recurrence.

\section{DISCUSSION}

Imatinib mesylate, a selective inhibitor of the tyrosine kinases, KIT, Bcr/Abl, PDGFRA, and PDGFRB, revolutionized the care of patients with GISTs and created a new paradigm in targeted cancer chemotherapy. As a neoadjuvant or adjuvant agent, however, the role of imatinib in the treatment of GISTs remains under investigation. In our three cases, neoadjuvant imatinib was able to reduce the size and extent of the initially unresectable locally advanced gastric GISTs. Only a few other reports have described the use of imatinib as a neoadjuvant 
Table 1. Pathologic changes before and after imatinib treatment

\begin{tabular}{ccccccccc}
\hline Case & Treatment & Morphology & Cellularity & \multicolumn{1}{c}{ Mitosis } & Necrosis & Hyalinization & Fibrosis & Immunoprofile \\
\hline \multirow{2}{*}{1} & Before & Spindle & High & $50 \sim 60 / 50 \mathrm{HPF}^{*} \mathrm{~s}$ & Absent & Absent & Absent & \\
& After & Spindle & Low & Rare & Absent & Marked & Absent & Same \\
2 & Before & Spindle & High & $5 / 20 \mathrm{HPF}^{*} \mathrm{~s}$ & Absent & Absent & Absent & Same \\
& After & Spindle & Low & Rare & Absent & Marked & Focal & Absent \\
3 & Before & Spindle & High & $10 / 20 \mathrm{HPF}^{*} \mathrm{~s}$ & Absent & Absent & Same \\
& After & Spindle & Low & Rare & Absent & Marked & Absent & Same \\
\hline
\end{tabular}

*High power field

treatment in GIST, including one report that described a patient with initially unresectable gastric GIST who underwent successful surgical resection after 6 months of imatinib treatment (12). The introduction of imatinib into clinical practice has caused a paradigm shift in the treatment of GIST, although clinical evidence is required to determine the optimal treatment strategy in patients with non-metastatic GIST. Resectable tumors should be treated with complete R0 resection, but unresectable locally advanced tumors may be downsized with imatinib and closely reevaluated for potential resectability. Our study and previous studies suggest that neoadjuvant imatinib could downsize primary unresectable tumors to resectable tumors, providing hopes for a cure in these settings.

In patients administered neoadjuvant imatinib for locally advanced GIST, questions remain regarding the length of imatinib therapy required prior to surgical resection. In the three patients described here, neoadjuvant imatinib therapy was administered for 7, 11, and 8 months, respectively. Interim data from a recent phase II randomized pivotal trial performed to investigate the outcome of imatinib therapy in patients with advanced GIST showed that the median time to response was 12 weeks. The study showed that $75 \%$ of patients responded to the drug at 23 weeks, and the median duration of response was 118 weeks. These findings suggest that neoadjuvant imatinib should be administered for at least $3 \sim 6$ months and surgical intervention, if possible, should be considered within 2 years after imatinib treatment (13). Thus, surgical intervention should be performed near the time of maximal tumor response, but before disease progression occurs.

The use of adjuvant imatinib as a standard therapy has not been established in patients with localized GISTs (2). Similarly, it has not been established whether a patient who underwent neoadjuvant therapy and complete tumor resection should receive postoperative imatinib therapy. For the first patient described in this study, imatinib therapy was maintained for 11 months postoperatively, because of liver metastases discovered prior to surgery. In the other two patients, who showed no evidence of distant metastases, imatinib therapy was not restarted after surgery. In all three patients, there was no evidence of tumor recurrence 22,15 , and 7 months, respectively, after surgery.

In one of our patients, a lesion highly suggestive of metastasis appeared on the liver after 5 months of imatinib treatment. This lesion, however, was totally necrotic and contained no viable cells at the time of surgery. It is possible that earlier stages of hepatic metastasis were not detected by CT scan at the time of diagnosis. This would occur if the metastasis was small and hypervascular, and therefore enhanced similarly to the surrounding hepatic parenchyma on portal-venous phase scans $(2,14)$.

Mutations involving exon 11 of the $c$-kit gene have been reported to be the most common in GIST, they also correlate with sensitivity to imatinib in vitro and in vivo (5). In the neoadjuvant setting, preoperative genotyping for kinase mutations may help predict clinical response to imatinib and could be used to develop a treatment plan (4). Mutations in exon 11 of the c-kit gene were detected in two of our patients (Cases 1 and 3), whereas no mutations in c-kit and PDGFRA were detected in our third patient. This tumor, however, showed good response to imatinib therapy.

When we reviewed the pathology in our three patients, we noted several features common to the imatinib treatment (Table 1). The initial biopsy specimens in all three patients revealed high cellularity and frequent mitoses. After treatment, however, all three resected specimens showed low cellularity, rare mitoses, and marked hyalinization. Except for the metastatic liver lesion, there was no evidence of necrosis. Other features, including the immunoprofile and cellular morphology, were identical to those of the pre-treatment biopsy.

Another significance of the neoadjuvant imatinib therapy for GISTs is that it may prevent marked loss of organ function in patients with extensive disease. These include gastric tumors with duodenal involvement, which may require Whipple's operation; esophageal tumors with esophagogastric involvement, which may require esophagogastric resection; and rectal GIST, in which the anal sphincter cannot be preserved. While we found that neoadjuvant imatinib could downsize tumor volume, it is not clear if it would be effective for all initially resectable tumors that do not appear to require such extensive surgery. Currently, neo-adjuvant imatinib is not recommended as a routine procedure because of the risk of primary resistance and the difficulties of evaluating tumor response (2).

The role of surgery in patients with metastatic GIST who responded well to initial imatinib treatment is another question to be answered. A recent study of initially unresectable GIST, in which most patients had metastases, suggested that surgical removal of the residual tumor after response to imatinib, when combined with continuous postoperative imatinib treatment, could allow for a complete remission and a prolonged durable remission in selected cases (15). But the issue remained unresolved.

To date, neoadjuvant imatinib treatment prior to optimal tumor resection is considered an experimental approach to be test- 
ed in prospective clinical studies (2). The survival benefit of this approach must await long-term follow-up of index cases and confirmation by clinical trials, including a current phase II trial (RTOG-S-0132). However, the three cases presented here suggest that neoadjuvant imatinib therapy combined with optimal evaluation and management can be a promising treatment strategy, and provides hope for a cure of patients with locally advanced GIST.

\section{REFERENCES}

1. Miettinen M, Majidi M, Lasota J. Pathology and diagnostic criteria of gastrointestinal stromal tumors (GISTs): a review. Eur J Cancer. 2002;38(Suppl 5):S39-51.

2. Blay JY, Bonvalot S, Casali P, Choi H, Debiec-Richter M, Dei Tos AP, et al. Consensus meeting for the management of gastrointestinal stromal tumors. Report of the GIST Consensus Conference of 20-21 March 2004, under the auspices of ESMO. Ann Oncol. 2005;16:566-78.

3. Dematteo RP, Heinrich MC, El-Rifai WM, Demetri G. Clinical management of gastrointestinal stromal tumors: before and after STI-571. Hum Pathol. 2002;33:466-77.

4. Heinrich MC, Corless CL, Duensing A, McGreevey L, Chen $\mathrm{CJ}$, Joseph N, et al. PDGFRA activating mutations in gastrointestinal stromal tumors. Science. 2003;299:708-10.

5. Heinrich MC, Corless CL, Demetri GD, Blanke CD, von Mehren $\mathrm{M}$, Joensuu $\mathrm{H}$, et al. Kinase mutations and imatinib response in patients with metastatic gastrointestinal stromal tumor. J Clin Oncol. 2003;21:4342-9.

6. Kim TW, Lee HN, Kang YK, Choe MS, Ryu MH, Chang HM, et al. Prognostic significance of c-kit mutation in localized gastrointestinal stromal tumors. Clin Cancer Res. 2004;10: 3076-81.

7. Verweij J, van Oosterom A, Blay JY, Judson I, Rodenhuis S, van der Graaf W, et al. Imatinib mesylate (STI-571 Glivec, Gleevec) is an active agent for gastrointestinal stromal tumours, but does not yield responses in other soft-tissue sarcomas that are unselected for a molecular target. Results from an EORTC Soft Tissue and Bone Sarcoma Group phase II study. Eur J Cancer. 2003;39:2006-11.

8. Verweij J, Casali PG, Zalcberg J, LeCesne A, Reichardt P, Blay JY, et al. Progression-free survival in gastrointestinal stromal tumours with high-dose imatinib: randomised trial. Lancet. 2004;364:1127-34.

9. Demetri GD, von Mehren M, Blanke CD, Van den Abbeele AD, Eisenberg B, Roberts PJ, et al. Efficacy and safety of imatinib mesylate in advanced gastrointestinal stromal tumors. N Engl J Med. 2002;347:472-80.

10. De Giorgi U, Verweij J. Imatinib and gastrointestinal stromal tumors: where do we go from here? Mol Cancer Ther. 2005; 4:495-501.

11. Ryu MH, Lee HN, Kim TW, Chang HM, Bang YJ, Kang WK, et al. Efficacy of imatinib mesylate and c-kit mutations in metastatic or unresectable GISTs. Ann Oncol. 2004;15:760 (abstr iii201).

12. Loughrey MB, Mitchell C, Mann GB, Michael M, Waring PM. Gastrointestinal stromal tumour treated with neoadjuvant imatinib. J Clin Pathol. 2005;58:779-81.

13. Blanke CD, Joensuu H, Demetri GD, Heinrich MC, Eisenberg $\mathrm{B}$, Fletcher J, et al. Outcome of advanced gastrointestinal stromal tumor (GIST) patients treated with imatinib mesylate: fouryear follow-up of a phase II randomized trial. ASCO Gastrointestinal Cancers Symposium Proceeding 2006;(abstr 7).

14. Ryu MH, Lee JL, Chang HM, Kim TW, Kang HJ, Sohn HJ, et al. Patterns of progression in gastrointestinal stromal tumor treated with imatinib mesylate. Jpn J Clin Oncol. 2006;36:17-24.

15. Rutkowski P, Nowecki Z, Nyckowski P, Dziewirski W, Grzesiakowska U, Nasierowska-Guttmejer A, et al. Surgical treatment of patients with initially inoperable and/or metastatic gastrointestinal stromal tumors (GIST) during therapy with imatinib mesylate. J Surg Oncol. 2006;93:304-11. 